\title{
AI Based Monitoring of Different Risk Levels in COVID-19 Context
}

\author{
César Melo $^{1, *,+(\mathbb{D}}$, Sandra Dixe ${ }^{2, *,+} \mathbb{D}$, Jaime C. Fonseca ${ }^{2} \mathbb{D}$, António H. J. Moreira ${ }^{3} \mathbb{D}$ and João Borges ${ }^{2,4} \mathbb{D}$ \\ 1 Engineering School, University of Minho, 4800-058 Guimarães, Portugal \\ 2 Algoritmi Center, University of Minho, 4800-058 Guimarães, Portugal; jaime@dei.uminho.pt (J.C.F.); \\ jpbsilva@algoritmi.uminho.pt (J.B.) \\ 3 2Ai, IPCA, School of Technology, 4750-810 Barcelos, Portugal; amoreira@ipca.pt \\ 4 Polytechnic Institute of Cávado and Ave, 4750-810 Barcelos, Portugal \\ * Correspondence: a74783@alunos.uminho.pt (C.M.); b12147@algoritmi.uminho.pt (S.D.); \\ Tel.: +351-917296547 (C.M.); +351-964079809 (S.D.) \\ + These authors contributed equally to this work.
}

Citation: Melo, C.; Dixe, S.; Fonseca, J.C.; Moreira, A.H.J.; Borges, J. AI Based Monitoring of Different Risk Levels in COVID-19 Context. Sensors 2021, 22, 298. https://doi.org/ $10.3390 /$ s22010298

Academic Editors: Elisenda Bonet and Xavier Paolo Burgos-Artizzu

Received: 2 November 2021

Accepted: 16 December 2021

Published: 31 December 2021

Publisher's Note: MDPI stays neutral with regard to jurisdictional claims in published maps and institutional affiliations.

Copyright: (c) 2021 by the authors. Licensee MDPI, Basel, Switzerland. This article is an open access article distributed under the terms and conditions of the Creative Commons Attribution (CC BY) license (https:// creativecommons.org/licenses/by/ $4.0 /)$.

\begin{abstract}
COVID-19 was responsible for devastating social, economic, and political effects all over the world. Although the health authorities imposed restrictions provided relief and assisted with trying to return society to normal life, it is imperative to monitor people's behavior and risk factors to keep virus transmission levels as low as possible. This article focuses on the application of deep learning algorithms to detect the presence of masks on people in public spaces (using RGB cameras), as well as the detection of the caruncle in the human eye area to make an accurate measurement of body temperature (using thermal cameras). For this task, synthetic data generation techniques were used to create hybrid datasets from public ones to train state-of-the-art algorithms, such as YOLOv5 object detector and a keypoint detector based on Resnet-50. For RGB mask detection, YOLOv5 achieved an average precision of $82.4 \%$. For thermal masks, glasses, and caruncle detection, YOLOv5 and keypoint detector achieved an average precision of $96.65 \%$ and $78.7 \%$, respectively. Moreover, RGB and thermal datasets were made publicly available.
\end{abstract}

Keywords: COVID-19; deep learning; supervised learning; object detection; keypoint detection

\section{Introduction}

In December 2019, an outbreak of pneumonia with unknown origins was reported in Wuhan, China. After conducting several tests around the associated virus, it was concluded to be a new variant of the existing coronavirus, associated with SARS-CoV. On 12 March 2020, the WHO declared a state of global emergency, considering it a pandemic, after nearly 125,000 cases were reported to be spread across more than 118 countries at that point. Since then, strict measures were implemented worldwide to contain the spread of the virus and reduce the chains of contagion, due to the virus' high level of transmissibility and inherently devastating effects, especially in people with chronic diseases, with weakened immune systems, and with those of older age. These measures severely affected all sectors, from the closure of the overwhelming majority of public establishments to bans on movement on public roads. The main symptoms of this disease are fever, cough, headaches, fatigue, and loss of taste, and its transmission through droplets released by the nose and mouth required rules of physical distancing and the mandatory use of masks in all activities that imply direct or indirect contact [1].

In this way, the need to develop advanced systems capable of monitoring people's behavior in an optimized way, especially in places that generate large concentrations of people in small areas, thus reducing as much as possible the spread of the virus within the community.

With the easing of restrictions, the levels of mobility and concentration of people, especially in public spaces and shopping areas, began to gradually increase again. However, 
the persistent presence of the virus means that behavior must still be moderate and adopt every precaution so that the number of infections and new infections remains at increasingly lower values to return normality in a manner as accelerated as possible. The fact that many people are asymptomatic to the disease also contributes to careless attitudes and negligent behavior, mainly associated with not wearing a mask. These behaviors and risk factors are imperative to monitor. Since this type of management is quite complicated to conduct in terms of human resources (e.g., at the entrances to shopping areas, where there are multiple entrance points and a large influx of people simultaneously), it is necessary to adopt methodologies that allow this monitoring to be performed in a more simple and optimized manner.

This article involves the presentation of the study and implementation of algorithms that allow, in real-time, the identification of risk factors and behaviors, such as the detection of the presence or absence of masks in people, as well as the precise measurement of their body temperature, to identify risk factors regarding possible cases of virus presence. This paper can be divided into two distinct modules: (A) detection of the presence or absence of masks on people in places where its use is mandatory, and secondly, (B) a punctual temperature measurement to detect situations where people are in a feverish state, one that represents a key symptom of the SARS-CoV-2 virus. Moreover, such algorithmic development is suitable to be implemented in an integrated system that allows to deploy a product in the market for monitoring purposes.

The main contributions of the paper are as follows:

1. A methodology for the generation of hybrid datasets with added masks on top of real samples from public datasets (Section 3.1.1);

2. An RGB dataset with added synthetic masks, on top of public datasets. MoLa RGB CovSurv [2] was made publicly available.

3. An IR dataset with information on the presence of the caruncle, masks, and glasses. MoLa IR CovSurv [3] was made publicly available.

4. State-of-the-art object detectors and keypoint face detectors were trained and evaluated, using a hyperparameter genetic search algorithm, i.e., Evolve. Considering the highest precision and lowest computational requirements, two models were selected. YOLOv5 small was the best choice for the RGB and IR mask and glasses detection. Moreover, a keypoint detector with a Resnet-50 backbone was selected for the caruncle detection in IR images.

Using these algorithms, we can implement them in an embedded system, and using the RGB cameras, we can install this as a monitoring system to assist with controlling the entrance of crowded establishments. Furthermore, it replaces the in-person task of measuring body temperature. The architecture description of the proposed solution can be consulted in Figure 1.

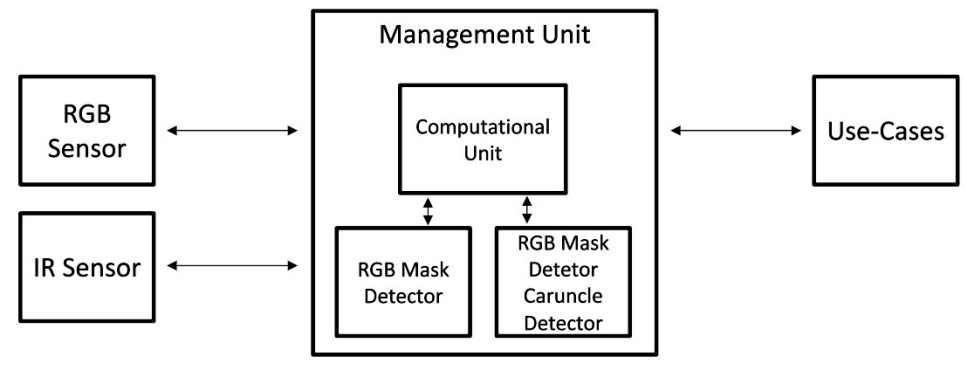

Figure 1. Architecture description of purposed solution.

The paper is organized as follows. Initially, the state-of-the-art is presented regarding deep-learning-based algorithmic solutions for the use-case at hand (i.e., RGB mask detection and IR keypoint detection). 
In the implementation section, for the RGB mask detection, a public dataset collection was made. Moreover, due to the lack of contextualized samples, a synthetic data generation toolchain was developed to generate the MoLa RGB CovSurv dataset [2].

For the IR algorithmic development, the same procedure was used, and publicly available datasets were used to create a pool of samples with extra label information (i.e., caruncle, mask, and glasses position). Moreover, a new MoLa IR CovSurv dataset [3] was formed.

Several evaluations were performed for RGB and IR detection use-cases using the generated datasets, where YOLOv5 was used as the main object detector, and a keypoint detector, based on the Resnet-50 backbone, was used for the caruncle detection.

Finally, results are presented and discussed, making it possible to select the best algorithms with the highest precision and lowest computational requirements.

Figure 2 summarizes the entire development pipeline of this article.

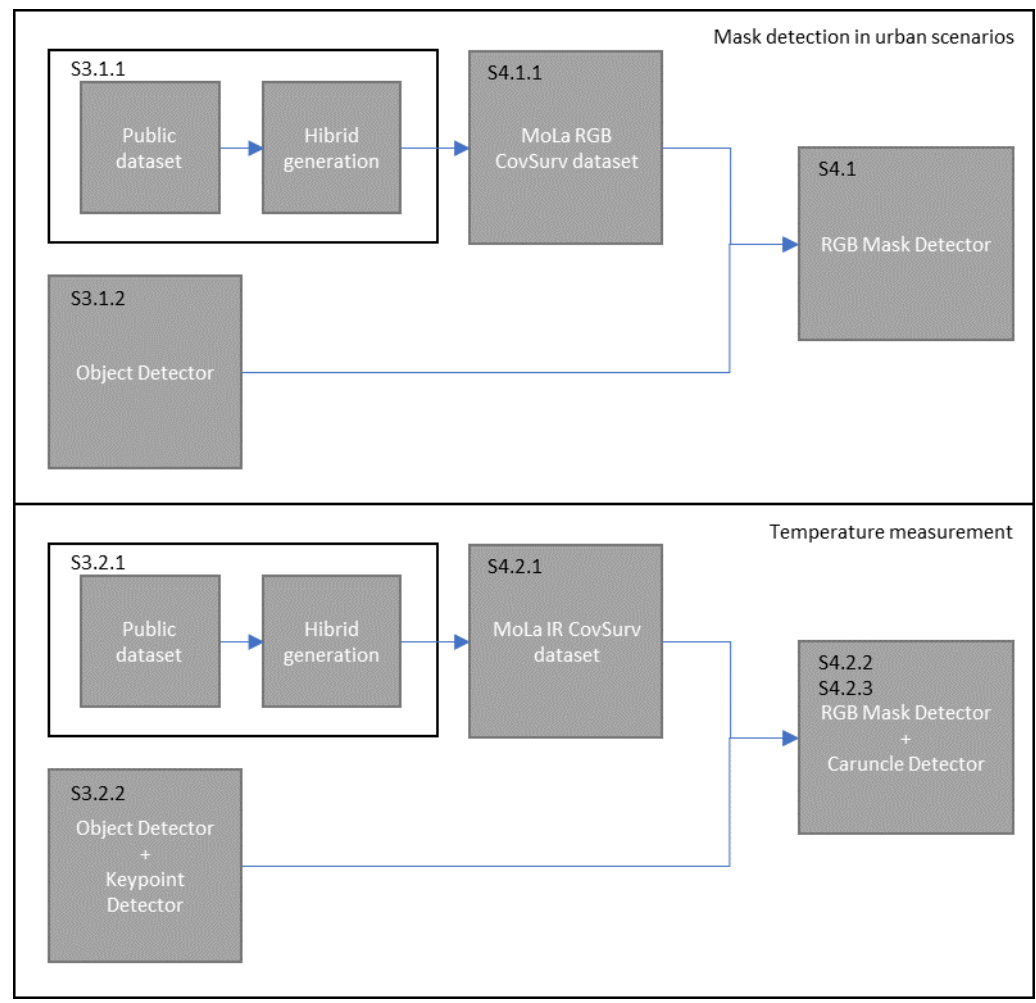

Figure 2. Two development pipelines are presented: (1) mask detection in urban surveillance; (2) temperature measurement. For each pipeline, 3 identical steps are made: (1) a toolchain for data generation is implemented (S3.1.1 and S3.2.1); (2) models are selected (S3.1.2 and S3.2.2); (3) datasets are generated (S4.1.1 and S4.2.1) to evaluate each model (S4.1, S4.2.2, and S4.2.3).

\section{Related Work}

Human mask detection in a surveillance scenario requires an approach similar to the ones used in object detection methodologies. There are several studies focused on object detection, which can be applied to various topics, and which can be an approach to consider in the task of mask detection. The authors [4-6] developed the R-CNN family of algorithms to detect different regions of interest in the image while using a CNN to classify the presence of the object in that region. More recently, the YOLO [7] object detection family presented as YOLOv2 [8], YOLOv3 [9], YOLOv4 [10], and YOLOv5 [11], provide a more accurate and faster method compared to the R-CNN family. Most recently, several object detection algorithms were used for the sole purpose of mask detection in a COVID-19 context. Jiang et al. [12] proposed a one-stage detector, achieving state-of-the-art results on a public face mask dataset. In the same context, Loey et al. [13] used YOLOv2 with a Resnet-50 backbone with two publicly available medical masks dataset, reaching an 
average precision of $81 \%$. Alternatively, the authors [14] used a single-shot detector with a MobileNetv2 backbone for the sole purpose of detecting masks in a surveillance scenario. Moreover, public datasets with real and synthetic samples were used for the algorithmic development, allowing to achieve $92.64 \%$ accuracy, with $64 \mathrm{~ms}$ of inference time.

For the detection of facial points, an important requirement for the detection of the caruncle location in human faces, state-of-the-art algorithms were developed. The first efficient algorithm for face detection in images was presented in 2001 by [15]. Later, in 2015, the authors [16] presented a cascaded CNN model, i.e., using 3 distinct CNNs (12-net, 24net, and 48-net), in which a gradual analysis of the image is performed, and initially, several small boxes are generated, which refer to certain facial elements; throughout the process, dimensional adjustments and calibrations are made until the face is identified as a whole. Sun et al. [17] presented an algorithm consisting of three levels of CNNs in cascade form for the detection of the five main facial points: Left-Eye Center (LE), Right-Eye Center (RE), Nose Tip (N), Left-Mouth Corner (LM) and Right-Mouth Corner (RM). It is a supervised approach, and when the bounding box of a face is provided, the location of the respective points is predicted. Haavisto et al. [18] presents a DBN-based algorithm to identify 15 facial points based on grayscale images. Longpre et al. [19] presented an approach to predict facial features in grayscale images. This algorithm consists of a mixture of convolutional layers based on the architectures of CNNs LeNet and VGG. Upon reception of an image, the goal is to return the coordinates $(x, y)$ of 30 facial points. Agarwal et al. [20] presented NaimishNet, an adaptation of LeNet architecture architecture for identifying facial features.

Several studies were already developed to monitor risk behavior in an attempt to mitigate the spread of COVID-19.

The author [21] proposed a monitoring and warning approach to respect social distancing (SD), relying on vision systems, and it was effective at preventing the spread of COVID-19 infectious disease. In this study, a real-time, vision-based system that can detect SD violations and send nonintrusive audio-visual cues using recent DL models is presented. A critical value of social density was defined, and they showed that the probability of occurrence of SD violation can be kept close to zero if the pedestrian density is kept below this value. The proposed system is also ethically fair: it does not record data or target individuals, and no human supervisor is present during operation. The proposed system was evaluated on real-world datasets.

The author [22] proposed a detection and diagnosis system using IoT-based smart glasses that can automatically and quickly detect COVID-19 from thermal images. The proposed design can perform face detection in case of suspected COVID-19 among crowds that have high body temperatures. The design will add information on the visited location of the suspected virus carriers through Google Location History (GLH) to provide reliable data on the detection process.

The authors $[23,24]$ evaluated the probability of the COVID-19 disease through sound analysis. Ref. [23] proposed the study of voice (speech) signal processing in the process of screening and early diagnosis of the COVID-19 virus, using Recurrent Neural Network (RNN), and more specifically, its well-known architecture, Long Short-Term Memory (LSTM), to analyze the acoustic characteristics of cough, breath, and voice of patients. The presented study shows a low accuracy in the voice test compared to that of the cough and breath sound samples. However, they highlight the possibility of increasing the accuracy of voice testing by expanding the dataset and targeting a larger group of healthy and infected people. Ref. [24] proposes a study that analyses cough sound. They present a reliable tool that can differentiate between different respiratory diseases, which is very relevant in the COVID-19 context.

The authors $[25,26]$ present DL approaches for detecting or not face masks on individuals. Ref. [25] proposes a system that restricts the growth of COVID-19 by tracking people not wearing a face mask in a smart city network where all public places are monitored by Closed Circuit Television (CCTV) cameras. While a person without a mask is detected, the corresponding authority is informed through the city network. It uses a DL architecture 
trained on a dataset consisting of images of people with and without masks collected from various sources. The trained architecture achieved $98.7 \%$ accuracy in distinguishing people with and without face masks using previously unseen test data. Ref. [26] proposes the implementation of a facial mask and social distancing detection model as an embedded vision system. The pretrained models such as MobileNet, ResNet classifier, and VGG are used in our context. People violating social distancing or not wearing masks were detected. After the implementation and deployment of the models, the selected one achieved a 100\% confidence index.

\section{Implementation}

\subsection{Urban Mask Detection}

\subsubsection{Synthetic Dataset Generation}

Since the amount of data used is also a very relevant factor for obtaining reliable and robust models, the need to develop a tool capable of generating synthetic images as a way to increase the available data arose. This tool was developed so that a wide variety of masks can be applied to public datasets, taking into account the position and orientation of faces, mask placement zone, and mask usage probability (as shown in Figure 3). For the generation of this synthetic dataset, it was decided to put synthetic masks on images of public datasets; thus, to perform this task, the first step is to find the faces of people in the images, and for this we used the method present in the open source library Dlib [27]. This method corresponds to a pretrained model based on HOG and SVM, which identifies faces in images, returning an object for each face detected. This object is of the type "rectangles", formed by two tuples representing, respectively, the coordinates of the upperleft and the lower-right corner points, which allow the formation of a rectangle around the detected face. Next, a function is applied that converts these two tuples into a bounding box. After extracting the bounding boxes associated with the faces present in the image, another pretrained method is used from the dlib library. Given the input image and the corresponding Region of Interest (ROI) (i.e., face detected by the previous method), the method tries to locate the face keypoints of interest within that region. In this tool, the detector estimates $682 \mathrm{D}$ points $(\mathrm{x}, \mathrm{y})$ associated with the other facial regions.

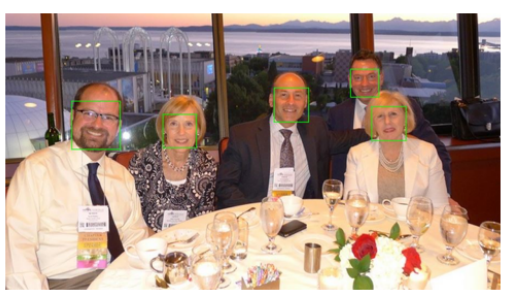

(a)

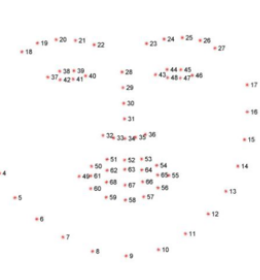

(b)

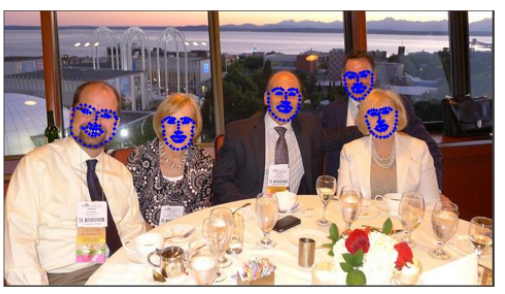

(c)

Figure 3. (a) Output of Get_Frontal_Face_Detected method of Dlib library. (b) Face location of each of shape_predictor_68_face_landmarks. (c) Output of pretrained model application shape_predictor_68_face_landmarks.

After the identification process of faces and respective facial keypoints, if more than 3 faces are identified in the image, $80 \%$ of the faces are randomly selected to be processed with the application of a synthetic mask, while the remaining $20 \%$ of the faces will remain unmasked. This methodology allows for an increase in the robustness of the algorithms to be trained, since, in this way, the final dataset will not be formed only by images with or without masks. For each of the faces to be masked, the type of mask is randomly selected, whether or not a texture will be applied and, if so, which texture will be used. The models of masks and some of the textures used can be seen in Figure $4 a, b$. 


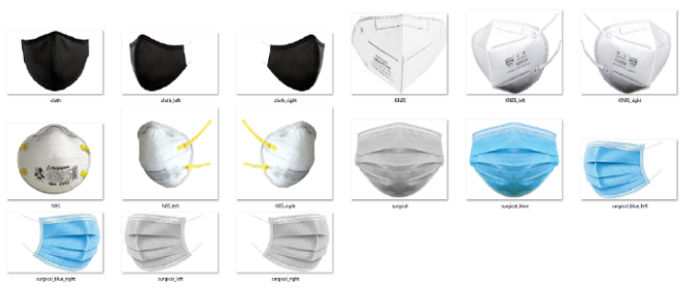

(a)
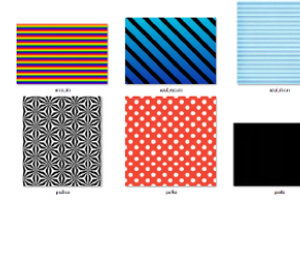

(b)

Figure 4. (a) Mask templates used when applying them synthetically to detected faces. (b) Set of examples among many mask textures applied with this tool.

Since not all the faces of the other datasets are in a frontal position in relation to the camera that captured them, affine transformations are performed on the other facial points obtained in the second step to understand which portion of the face is visible. For this reason, and as shown in Figure 4a, different perspectives are available for each mask model, according to the facial visibility. The samples in which this tool was used belong to the already existing datasets listed in the Table 1 . The use of different datasets, in addition to increasing the number of samples, allows to enhance the algorithms to be trained, since there are samples with different quality levels, occlusion, luminosity, background, and number of people.

Table 1. Datasets where proposed tool was applied.

\begin{tabular}{cl}
\hline Dataset & \multicolumn{1}{c}{ Description } \\
\hline Celeba ([28]) & More than 200,000 images of faces. Images of just one person. \\
\hline Coco ([29]) & $\begin{array}{l}\text { More than 320,000 images and more than 91 different objects, widely used } \\
\text { for object detection tasks. We only used images where people are present } \\
\text { in the most varied environments and contexts. }\end{array}$ \\
\hline Helen ([30]) & It consists of 2330 images of one or several people. \\
\hline IMM ([31]) & Consisting of 240 single-face images of 40 different people. \\
\hline Wider ([32]) & Over 32,000 images with different levels of scale and occlusion. \\
\hline Group Images ([33]) & More than 5000 images of groups of people. \\
\hline
\end{tabular}

After performing some tests of the tool on the different samples present in the datasets listed in Table 1, it was concluded that the method associated with the dlib library for detecting facial points was not very effective when the faces were not at a relatively frontal angle; the identification of the faces happened, but they were considered as if they were at a frontal angle, which led to poor applications of the synthetic masks. An individual analysis was made of all the samples used to discard those in which the tool did not work as expected. For these incorrect samples, the respective annotations of the other datasets were used, in the cases where they were provided, to obtain the exact facial points for the correct application of the synthetic mask. In situations where the samples were not accompanied by annotations, another pretrained model was used, called MobileFaceNet [34], capable of predicting more accurately the same 68 points associated with each face, even if they are not visible in the image. Some final results obtained from the use of the tool can be verified in the Figure 5.

For this dataset, two classes were considered, "With Mask" and "Without Mask", to which IDs were assigned " 0 " and " 1 ", respectively. As the object to be identified is always the face of a person, regardless of the presence or absence of mask, the labels associated to each image were based on the information provided by the method get_frontal_face_detector applied in the first stage of the tool for applying synthetic masks, which is responsible for identifying the faces present in a sample from the return of the coordinates delimiting each of the objects found. Thus, it was only necessary to normalize 
this data according to the dimensions of each image. Finally, the MoLa RGB CovSurv dataset was generated, and made publicly available [2].

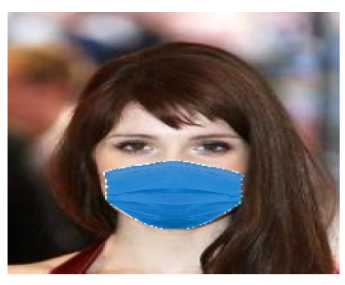

(a)

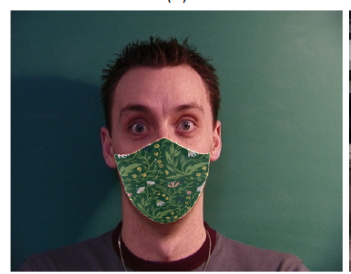

(d)

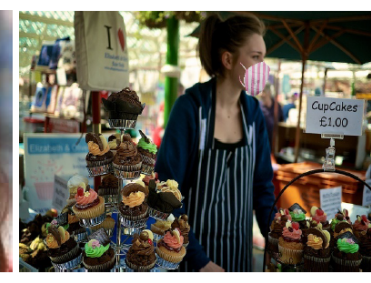

(b)

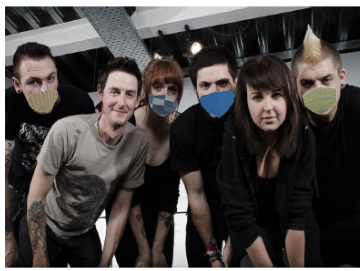

(e)

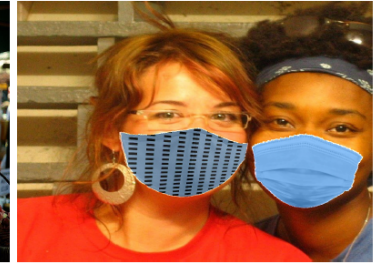

(c)

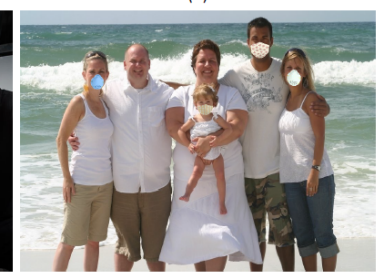

(f)

Figure 5. Examples of samples subjected to application of synthetic masks from MobileFaceNet tool. (a) Dataset Celeba. (b) Dataset Coco. (c) Dataset Helen. (d) Dataset IMM. (e) Dataset Wider. (f) Dataset Group Images.

The number of labels associated with class " 0 " (face with mask) is approximately 55,000 and class " 1 " (face without mask) is 20,000 . These labels are used for training the selected algorithms. This imbalance is due to the fact that with the tool presented in Section 3.1.2, masking was applied to $80 \%$ of the identified faces in each sample with more than three identified in each sample, since, in real situations, the tendency is the presence of a large majority of masks.

\subsubsection{Model}

To perform the mask detection task the YOLOv5 family will be used, specifically the small, medium, large, and extra-large models, which differ in the depth of their layers, realtime performance, and detection accuracy. Input image resolution was fixed at a $512 \times 512$, with two classes output. Anchor boxes will be calculated automatically for the training dataset.

\subsection{IR Temperature Detection}

\subsubsection{Dataset Generation}

Another risk factor that may reflect the presence of the SARS-CoV2 virus is high body temperature, which usually indicates a feverish state. Similarly to the previous chapter, the need arises to collect samples that will be the base for training algorithms capable of identifying, in a thermographic context, the presence of masks, goggles, and facial areas where temperature measurement is carried out in a more reliable way. In this case, it corresponds to the tear area present in each eye of the human being (more specifically, the caruncle area [35]). These samples were obtained from public datasets, and also from samples generated in laboratory. The latter were based on the availability of 30 volunteers to perform a series of recordings in different scenarios. These recordings, using a thermographic camera (FLIR ADK [36]), consisted of the continuous movement of approaching the camera up to a $30 \mathrm{~cm}$ distance, followed by the approximation of the face, as a way of making the areas associated with the caruncle visible, for later analysis and creation of labels for algorithm training. In Table 2 are described the existing datasets formed by the thermographic images used in the training of the selected algorithms, and that represent a large portion of the final dataset generated. In Figure 6, it is possible to see some samples of these same datasets. 
Table 2. Thermographic datasets collected for training of mask, goggles, and caruncle zone detection algorithms.

\begin{tabular}{ll}
\hline \multicolumn{1}{c}{ Dataset } & \multicolumn{1}{c}{ Description } \\
\hline $\begin{array}{l}\text { IIITD In and Beyond Visible Spectrum Disguise } \\
\text { Database ([37]) }\end{array}$ & $\begin{array}{l}\text { Thermographic images of } 75 \text { different people, with and without } \\
\text { glasses, with and without mask. }\end{array}$ \\
\hline UL-FMTV Database ([38]) & $\begin{array}{l}\text { Consisting of 18,210 thermographic images of samples of people with } \\
\text { and without glasses. }\end{array}$ \\
\hline Terravic Facial Infrared Database ([39]) & It consists of 22,784 thermographic images of 18 different people. \\
\hline IRIS Database ([40]) & $\begin{array}{l}\text { Consisting of thermographic images of 31 different people, with } \\
\text { different lighting, expressions, and pose conditions. }\end{array}$ \\
\hline
\end{tabular}
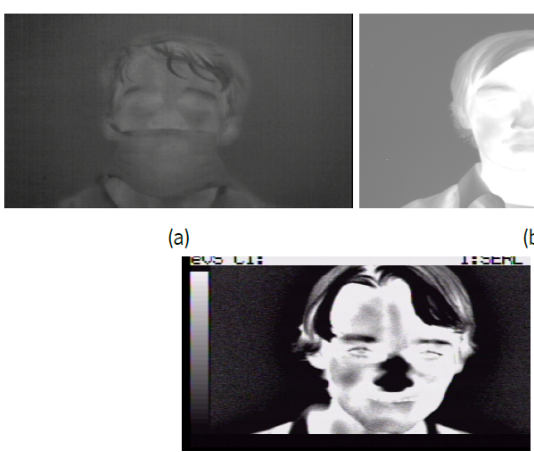

(d)
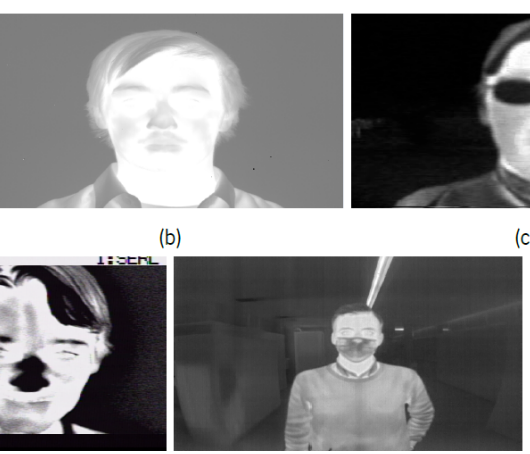

(e)

Figure 6. Sample examples of other thermographic datasets used for facial point detection task. (a) IIITD In and Beyond Visible Spectrum Disguise Database. (b) UL-FMTV Database. (c) Terravic Facial Infrared Database. (d) IRIS Database. (e) Sample collected in laboratory.

For the mask and glasses detection component, the labels go through only the location of the face in the image, whose classes to be identified by the selected object detection algorithms are presented in Table 3. For the detection of the facial keypoints of interest, the labels also included the identification of the face in each sample, with the addition of the location of the two points associated with the caruncle of both eyes.

Table 3. Classes to be identified and their IDs, in the masks and goggles detection task, in the thermography component.

\begin{tabular}{cc}
\hline Classe & ID \\
\hline Face_Mask_Eyes & 0 \\
\hline Face_Mask_NoEyes & 1 \\
\hline Face_NoMask_Eyes & 2 \\
\hline Face_NoMask_NoEyes & 3 \\
\hline
\end{tabular}

The necessary labels were generated in a semiautomatic labeling process. In the first stage, all the samples that make up the thermographic dataset were subjected to passing through the pretrained models that constitute the first two steps used for the development of the tool responsible for applying synthetic masks, get_frontal_face_detector and shape_predictor_68_face_landmarks, whose functions include the identification of faces and the location of the 68 points associated with the facial regions. As we are dealing with thermographic images and some of them have masks and/or glasses present, these models presented certain difficulties in identifying the desired information in most of the samples constituting the dataset. Thus, for the samples with satisfactory results, the information returned by the models was converted to the formats used by the different algorithms to associate the labels to the respective images. In situations where the results did not meet expectations, manual labeling was performed using the online tool V7Darwin [41] and 
the labels to be identified in each image were generated one by one. Figure 7 shows two examples where it is possible to consult the labels obtained automatically and manually. With attention to Figure 7a, although the facial points associated with the mandible and mouth region were poorly identified since a mask is present, the bounding box of the face present as well as the points of interest (left- and right-caruncles) were well identified. In this case, this information was taken into consideration for label formation. Finally, the MoLa IR CovSurv dataset was generated and made publicly available [3]. Figure 8 shows the number of samples that make up the generated dataset, associated with each of the classes to be identified. The great unbalance between class 1 , associated with people wearing a mask and wearing glasses, in relation to the other classes, is due to the fact that both in the existing datasets collected, as well as in the people who volunteered to make laboratory recordings, the presence of glasses was quite scarce. Classes 0 and 1 come essentially from the recordings made, where the presence of mask predominates, while classes 2 and 3 belong mostly to the datasets presented in Table 2 .

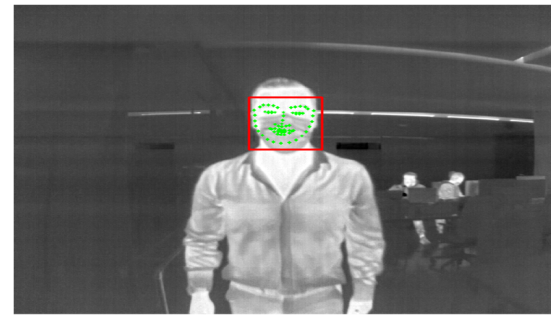

(a)

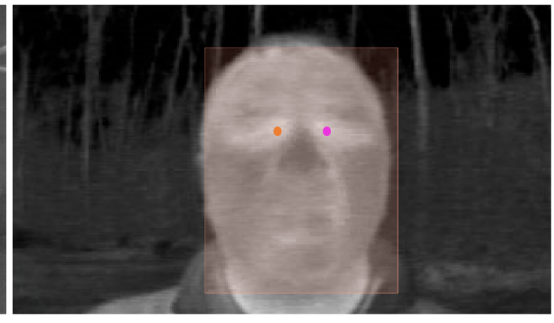

(b)

Figure 7. Examples of samples where semiautomatic label technique was applied. (a) Automatic Labeling. Red rectangle refers to bounding box of identified face, and green dots to respective facial marks. (b) Manual labeling. Orange rectangle is associated to identified face, while orange and violet dots refer, respectively, to points that identify right- and left-caruncles.

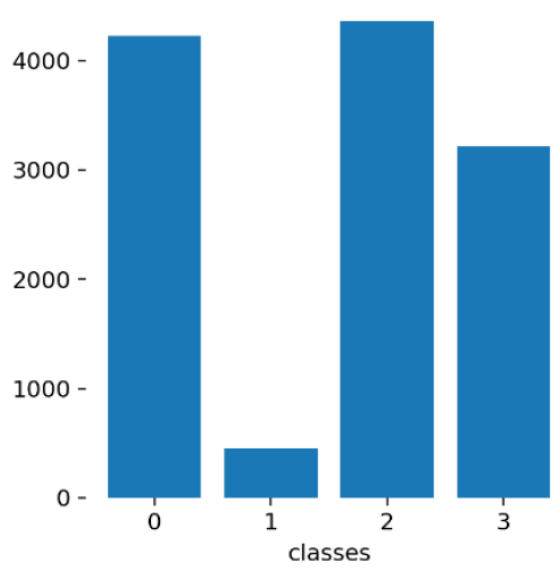

Figure 8. Number of labels associated to different classes existing in mask and glasses detection task. Different classes can be consulted in Table 3.

\subsubsection{Model}

For the detection of masks and glasses in thermographic samples, object detection models are highly contextualized. As such, YOLOv5 was selected for the evaluations, with an input image resolution fixed at $512 \times 512$, and four classes output. For the caruncle detection a keypoint detector, Ref. [42] was selected, with different backbones available (i.e., Resnet-\# and HrNetv2_w\#), input image resolution was also fixed at $512 \times 512$, with two heatmaps output. 


\section{Experiments and Results}

The objective of this section is to evaluate the algorithms used for the detection tasks we have set ourselves. The algorithms trained for these tasks are the variants of the YOLOv5 architecture (Section 3.1.2) and the keypoint detection algorithms (Section 3.2.2), whose backbones correspond to architectures of the CNNs Resnet and HRNetv2 families. All these tests were performed on a server with an Intel(R) Xeon(R) Gold 6140 CPU 2.30Ghz processor, 128GB RAM, and NVIDIA Tesla V100-PCIE-16GB computing GPU.

\subsection{RGB Detection}

\subsubsection{Dataset}

For the RGB detection evaluations, MoLa RGB CovSurv dataset was used. Table 4 shows the description of each subset of the dataset used for mask detection.

Table 4. Description of each subset of dataset used to detect presence or absence of masks.

\begin{tabular}{cl}
\hline Subset of Dataset & \multicolumn{1}{c}{ Description } \\
\hline Training & $\begin{array}{l}\text { Images generated from the datasets presented in the Table 1. Consisting of a } \\
\text { total of 40,972 samples. }\end{array}$ \\
\hline Validation & $\begin{array}{l}\text { It consists of 758 real samples of people with and without mask. The dataset } \\
\text { used is presented in [43]. }\end{array}$ \\
\hline Test & $\begin{array}{l}\text { It consists of 3441 real samples of people with and without mask. The dataset } \\
\text { used is presented in [44]. }\end{array}$ \\
\hline
\end{tabular}

The approach presented in Table 4 is unbalanced in quantitative terms and is justified by the fact that the training images are generated in a synthetic way, so the best way to obtain more reliable metrics is to validate and test the model with totally realistic images.

\subsubsection{RGB Mask Detection}

To reach the best precision model, the four YOLOv5 models were evaluated in an iterative way: firstly, an Evolve technique was used to find the best hiperperameters, and secondly, the obtained values in the final training were utilized (i.e., E\#->E\#.1). Table 5 shows the evaluations performed. The values of the finetune hyperparameters used in tests E1, E2, E3, and E4 are predefined by the authors [11], obtained after performing a medium model training of 50 epochs on the COCO dataset. To compare with our YOLOv5 family approach, we tested the face mask detection method, presented in [45], with our test dataset [44], to compare the obtained metrics of both models. This method uses an SSD framework, and it was trained on the dataset presented in [32]. To increase the speed of the network, the authors used a lite backbone with only 8 convolutional layers. Like our YOLOv5 models, the goal of this method is to detect faces and determine if they are wearing masks.

Table 5. Tests performed using four object detection algorithms selected for mask detection task.

\begin{tabular}{ccccccc}
\hline Trial & Dataset & Modelo & Epochs & Evolve & Evolve-Epochs & Hyperparameters \\
\hline E1 & $30 \%$ Train, 30\% Valid & YOLOv5s & 3 & Yes & 100 & FineTuneParams \\
\hline E1.1 & $100 \%$ Train, 100\% Valid & YOLOv5s & 25 & No & 0 & E1_EvolveParams \\
\hline E2 & $30 \%$ Train, 30\% Valid & YOLOv5m & 3 & Yes & 100 & FineTuneParams \\
\hline E2.1 & $100 \%$ Train, 100\% Valid & YOLOv5m & 25 & No & 0 & E2_EvolveParams \\
\hline E3 & $30 \%$ Train, 30\% Valid & YOLOv5l & 3 & Yes & 100 & FineTuneParams \\
\hline E3.1 & $100 \%$ Train, 100\% Valid & YOLOv5l & 25 & Não & 0 & E3_EvolveParams \\
\hline E4 & $30 \%$ Train, 30\% Valid & YOLOv5x & 3 & Yes & 100 & FineTuneParams \\
\hline E4.1 & $100 \%$ Train, 100\% Valid & YOLOv5x & 25 & No & 0 & E4_EvolveParams \\
\hline
\end{tabular}


Table 6 presents the metrics obtained from performing the trials presented in Table 5, based on the hyperparameters obtained by the "Evolve" method (see Table 7). The average accuracies of each class can be calculated based on the analysis of the precision-recall curve, presented in Figure 9 for each of the models (YOLOv5 and FaceMaskDetection-SSD).

Table 6. Metrics obtained from trials in Table 5. Bold lines represent results for the selected model.

\begin{tabular}{ccccc}
\hline \multicolumn{5}{c}{ YOLOv5 } \\
\hline Hyperparameter & Small (E1.1) & Medium (E2.1) & Large (E3.1) & Extra-Large (E4.1) \\
\hline Precision & $\mathbf{7 1 . 0 1 \%}$ & $73.34 \%$ & $82.77 \%$ & $66.18 \%$ \\
\hline mAP_0.5 & $\mathbf{8 2 . 3 8 \%}$ & $81.92 \%$ & $83.25 \%$ & $81.43 \%$ \\
\hline mAP_0.5:0.95 & $\mathbf{4 6 . 5 2 \%}$ & $46.17 \%$ & $45.96 \%$ & $45.05 \%$ \\
\hline Recall & $\mathbf{8 1 . 9 8 \%}$ & $81.19 \%$ & $77.68 \%$ & $81.67 \%$ \\
\hline
\end{tabular}

Table 7. Values assigned to main hyperparameters after performing Evolve technique on YOLOv5 models for trials in Table 5.

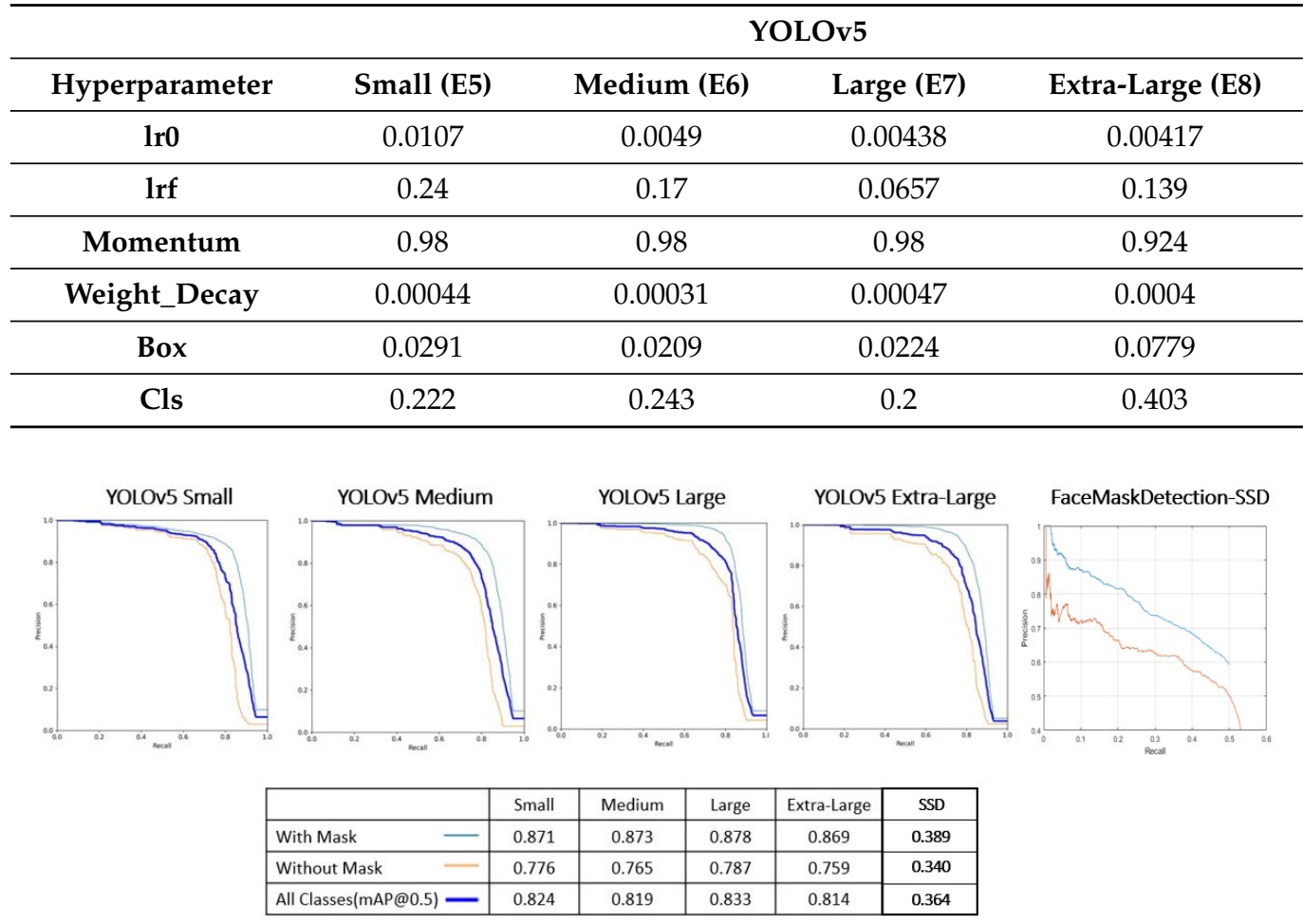

Figure 9. Presentation of Precision-Recall curves of models. In legend of each model, you can see average accuracies obtained for each class.

\subsection{IR Detection}

\subsubsection{Dataset}

For the IR detection evaluations, MoLa IR CovSurv dataset was used. Table 8 presents the training, validation, and test subsets that form the final dataset used for training the mask and glasses object detection algorithms. 
Table 8. Description of each subset of dataset used for mask and goggle detection in a thermographic context.

\begin{tabular}{cc}
\hline Subset of Dataset & Description \\
\hline Train & 12,254 samples \\
\hline Validation & 3501 samples \\
\hline Test & 1750 samples \\
\hline
\end{tabular}

For the keypoint detection algorithms associated with the human caruncle, the dataset used in this task is constituted by a 70\% fraction of the MoLa IR CovSurv dataset, used for mask and glasses detection, presented in Table 8, from which only the samples that present visible caruncles were selected, regardless of the presence or not of mask. Table 9 describes the subsets of this same dataset.

Table 9. Description of each subset of dataset used for detection of the human caruncle area in thermographic context.

\begin{tabular}{cl}
\hline Subset of Dataset & Description \\
\hline Train & 8602 samples \\
\hline Validation & 2456 samples \\
\hline Test & 1229 samples \\
\hline
\end{tabular}

\subsubsection{Mask and Glasses Detection}

As in Section 4.1.2, for the detection of masks and glasses in IR images, the four YOLOv5 models were evaluated in an iterative methodology. Table 10 describes the different trials performed to obtain the models used in the mask and goggles detection component. For tests E5, E6, E7, and E8, the hyperparameters presented by the authors [11] were used.

Table 10. Tests performed for four object detection algorithms selected for masks and glasses detection task using thermographic dataset.

\begin{tabular}{ccccccc}
\hline Trial & Dataset & Model & Epochs & Evolve & Evolve-Epochs & Hyperparameters \\
\hline E5 & $100 \%$ Train, $100 \%$ Valid & YOLOv5s & 3 & Yes & 100 & FineTuneParams \\
\hline E5.1 & $100 \%$ Train, 100\% Valid & YOLOv5s & 20 & No & 0 & E5_EvolveParams \\
\hline E6 & $100 \%$ Train, 100\% Valid & YOLOv5m & 3 & Yes & 100 & FineTuneParams \\
\hline E6.1 & $100 \%$ Train, 100\% Valid & YOLOv5m & 20 & No & 0 & E6_EvolveParams \\
\hline E7 & $100 \%$ Train, 100\% Valid & YOLOv51 & 3 & Yes & 100 & FineTuneParams \\
\hline E7.1 & $100 \%$ Train, $100 \%$ Valid & YOLOv5l & 20 & No & 0 & E7_EvolveParams \\
\hline E8 & $100 \%$ Train, $100 \%$ Valid & YOLOv5x & 3 & Yes & 100 & FineTuneParams \\
\hline E8.1 & $100 \%$ Train, 100\% Valid & YOLOv5x & 20 & No & 0 & E8_EvolveParams \\
\hline
\end{tabular}

Table 11 presents the metrics obtained from performing the tests presented in Table 10, based on the hyperparameters obtained by the "Evolve" method (see Table 12). The average accuracies of each class can be calculated based on the analysis of the precision-recall curve, presented in Figure 10 for each of the models (YOLOv5). 
Table 11. Metrics obtained from trials in Table 10. Bold lines represent results for selected model.

\begin{tabular}{ccccc}
\hline \multicolumn{5}{c}{ YOLOv5 } \\
\hline Metrics & Small (E5.1) & Medium (E6.1) & Large (E7.1) & Extra-Large (E8.1) \\
\hline Precision & $\mathbf{8 1 . 8 6 \%}$ & $83.15 \%$ & $85.23 \%$ & $91.19 \%$ \\
\hline mAP_0.5 & $\mathbf{9 6 . 6 5 \%}$ & $96.43 \%$ & $96.53 \%$ & $98.34 \%$ \\
\hline mAP_0.5:0.95 & $\mathbf{7 1 . 3 3 \%}$ & $72.85 \%$ & $72.60 \%$ & $75.99 \%$ \\
\hline Recall & $\mathbf{9 6 . 3 9 \%}$ & $95.76 \%$ & $96.78 \%$ & $97.97 \%$ \\
\hline
\end{tabular}

Table 12. Values assigned to main hyperparameters after performing Evolve technique of the YOLOv5 models, for the trials in Table 10.

\begin{tabular}{ccccc}
\hline & \multicolumn{5}{c}{ YOLOv5 } \\
\hline Hyperparameter & Small (E5) & Medium (E6) & Large (E7) & Extra-Large (E8) \\
\hline lr0 & 0.0033 & 0.00334 & 0.00376 & 0.00424 \\
\hline lrf & 0.114 & 0.1 & 0.121 & 0.141 \\
\hline Momentum & 0.972 & 0.98 & 0.968 & 0.959 \\
\hline Weight_Decay & 0.00037 & 0.00031 & 0.00028 & 0.00026 \\
\hline Box & 0.0385 & 0.237 & 0.0269 & 0.0273 \\
\hline Cls & 0.299 & 0.265 & 0.214 & 0.2 \\
\hline
\end{tabular}
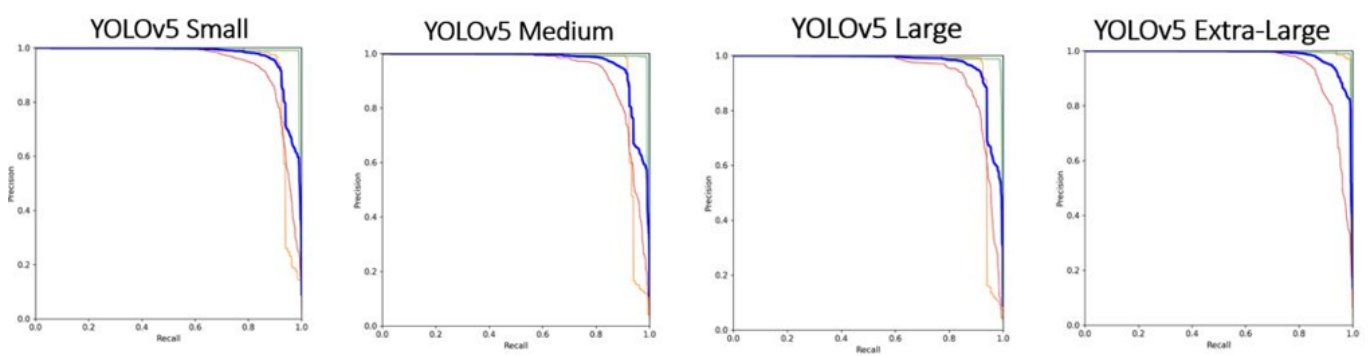

\begin{tabular}{|l|c|c|c|c|}
\hline & Small & Medium & Large & Extra-Large \\
\hline Face Mask Eyes & 0.996 & 0.996 & 0.996 & 0.996 \\
\hline Face Mask NoEyes & 0.942 & 0.936 & 0.941 & 0.994 \\
\hline Face NoMask Eyes - & 0.990 & 0.991 & 0.990 & 0.993 \\
\hline Face NoMask NoEyes - & 0.938 & 0.934 & 0.935 & 0.950 \\
\hline All classes (mAP@0.5) - & 0.966 & 0.964 & 0.965 & 0.983 \\
\hline
\end{tabular}

Figure 10. Presentation of Precision-Recall curves of models. In the legend of each model, you can see average accuracies obtained for each class.

\subsubsection{Caruncle Detection}

Table 13 shows the different tests performed for the task of detecting the area of the caruncle area of each eye. For the same model, 6 backbones were evaluated to select the highest performing and lowest computational requirements model. 
Table 13. Trials performed for different algorithms selected for human caruncle detection task.

\begin{tabular}{cccc}
\hline Trial & Backbone & Epochs & Learning Rate \\
\hline E9 & Resnet-50 & 5 & 0.002 \\
\hline E10 & Resnet-101 & 5 & 0.002 \\
\hline E11 & Resnet-152 & 5 & 0.002 \\
\hline E12 & HrNetv2_w18 & 5 & 0.002 \\
\hline E13 & HrNetv2_w32 & 5 & 0.002 \\
\hline E14 & HrNetv2_w48 & 5 & 0.002 \\
\hline
\end{tabular}

Table 14 shows the results of the tests of Table 13. Normalized Mean Error (NME) is associated to the average error of the distance between the estimated points and the ground-truth points previously labeled, relative to the training samples. The "Inference Time" column refers to the time that each algorithm needs to analyze an image from the test dataset, presented in seconds. The column "Precision" refers to the accuracy of the algorithm on the test dataset within a margin of 5 pixels; that is, if the distance between the facial points calculated by the algorithm and the previously labeled facial points (groundtruth) is smaller than 5 pixels, a correct prediction is considered. Its calculation is given by the ratio between predictions considered true positives and all predicted positives.

Table 14. Results obtained for each trial performed in human caruncle detection task. Bold lines represent best selected results for each model.

\begin{tabular}{ccccc}
\hline Trial & NME & Inference Time (seconds) & Precision & Average Error (pixels) \\
\hline E9 & $\mathbf{0 . 0 9 4 5}$ & $\mathbf{0 . 0 2 4}$ & $\mathbf{7 8 . 6 8 \%}$ & $\mathbf{3 . 8 9}$ \\
\hline E10 & 0.1012 & 0.04 & $78.44 \%$ & 3.86 \\
\hline E11 & 0.1029 & 0.057 & $77.46 \%$ & 3.92 \\
\hline E12 & 0.0905 & 0.107 & $82.1 \%$ & 3.35 \\
\hline E13 & 0.0975 & 0.103 & $79.78 \%$ & 3.77 \\
\hline E14 & 0.0971 & 0.104 & $79.05 \%$ & 3.66 \\
\hline
\end{tabular}

\section{Discussion}

In Section 4.1, the object detection algorithms, YOLOv5 family and FaceMaskDetectionSSD, are evaluated ore precisely to detect the presence or absence of masking. Although all the algorithms of the YOLOv5 family presented good results, the method to be used for the mask detection task is the Small model of the YOLOv5 architecture. This choice is justified by the fact that the different metrics obtained do not change substantially, since more layers were added along the remaining deeper models, and the task does not present a high degree of complexity since it is intended to detect only two distinct classes (with or without mask). Considering the inference times obtained are: $0.032 \mathrm{~s}$ for the Small model, $0.045 \mathrm{~s}$ for the Medium model, 0.062 for the Large model, and $0.089 \mathrm{~s}$ for the Extra-Large model. Thus, the best choice was to select the lightest model (Small), with $82.38 \%$ of mAP_0.5. Figure $11 \mathrm{~b}$ shows qualitative results obtained on different samples, based on the inference of the selected model. The FaceMaskDetection-SSD method shows a 36.4\% of mAP_0.5 when inferred on our test dataset. This may be because the model was trained on 7971 samples, which is a significantly lower number than our dataset. Hence, its inference capability on our test dataset is much lower. Furthermore, the FaceMaskDetection-SSD model has a lower complexity than our lighter model, YOLOv5s, with $1.01 \mathrm{M}$ and $1.9 \mathrm{M}$ parameters, respectively. Section 4.2 presents models capable of detecting the facial points of interest (using a thermographic camera) to be able to carry out effective temperature measurements as a way to screen for the potential presence of the SARS-CoV2 virus. 
This task is composed of two distinct steps: in the first step, and given that temperature measurements are not possible with the presence of glasses, object detection algorithms capable of detecting not only the presence of this object, but also the presence of masks were implemented (Section 4.2.2). In the second step, for the glasses and mask detection component, the algorithms forming the YOLOv5 architecture were selected, while for the face points detection component (Section 4.2.3), algorithms whose Backbones are made up of CNNs that are part of the Resnet and HrNetv2 architectures were selected. The results obtained by the different algorithms for both steps are quite satisfactory in the sense that these results experience practically no improvement with the use of deeper algorithms, since the number of classes and face points to be identified is quite low, in conjunction with the use of a highly uniform dataset whose samples are quite similar. Since the goal was to achieve high precision and low computational requirements, this led to the choice of the Small model for the glasses and mask detection aspect (corresponding to E5.1, with a precision of $81.86 \%$ and an inference time equal to the selected model for mask detection, $0.032 \mathrm{~s}$ ), and the model with Backbone Resnet-50 (corresponding to E9, with a precision of $78.68 \%$ and an inference time of $0.024 \mathrm{~s}$ ). Figure $11 \mathrm{a}$ shows qualitative results from the inference of the algorithms chosen for both tasks.

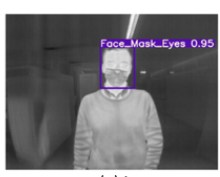

(a) I

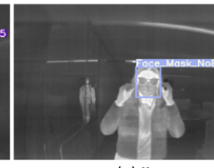

(a) II

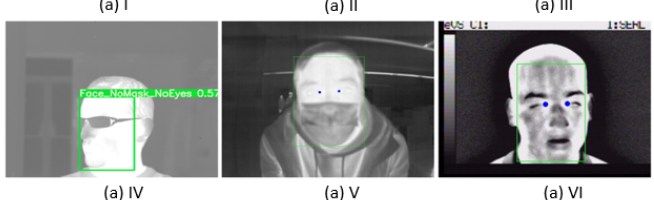

(a)

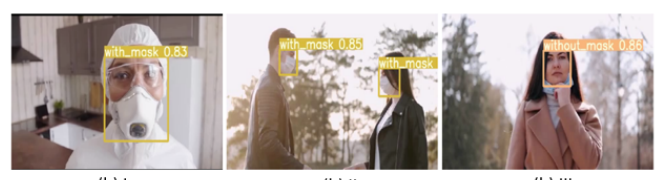

(b) I

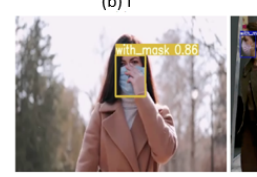

(b) IV

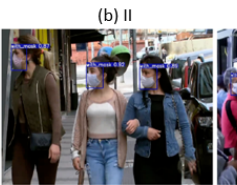

(b) $\mathrm{V}$

(b)

Figure 11. (a-I - a-VI) Examples of results obtained in images, from inference of selected algorithms for mask and glasses detection components and detection of zone corresponding to human caruncle. (b-I - b-VI) Examples of results obtained in images, from inference of selected YOLOv5 algorithm for mask detection.

\section{Conclusions}

This article presents a system capable of detecting behaviors and risk factors of people within the scope of the COVID-19 pandemic, and more specifically, the implementation of algorithms for the detection of masks in public spaces, as well as the punctual execution of temperature measurements for the detection of possible cases of fever. Initially, a search was carried out associated with the existing state-of-the-art algorithms suitable for performing the proposed tasks. The selected algorithms belong to the themes of object detection and Keypoint Detection. The first task was mask detection in RGB images. As a basis for training the selected algorithms in this component, it was necessary to create a dataset and generate the respective labels. Regarding the dataset and given that the number of existing samples in this area is still scarce, a tool capable of applying synthetic masks to RGB images was developed, using pretrained models capable of locating the faces present and their respective facial points. Based on this information, a mask is subsequently applied within the existing types and textures to the facial points where it should be placed. The labels associated with this dataset were automatically sourced from the pretrained models used. Subsequently, using this dataset, multiple algorithms based on the YOLOv5 architecture were evaluated. After the training and respective evaluation of the results obtained, all models obtained good results, however, the Small model was the selected one (with a 
precision of $71.01 \%$ ). This choice is justified because the obtained metrics are very similar despite the use of different and deeper models, mainly due to the fact that the required degree of complexity is not high because it is only intended to detect two different classes. Another reason is the balance between precision and real-time performance of the Small model regarding the other tested models.

For the temperature measurement component, it was also necessary to create a dataset consisting of thermographic images and generate the respective labels. In this case, algorithms were implemented both for mask and goggles detection, and for the detection of facial points associated with the human caruncle area, where the temperature measurement is performed with greater accuracy. The labels were originated in a semiautomatic way, i.e., based on the pretrained models enunciated in the previous task, as well as from manual labeling, image by image. For the mask and glasses detection task, the models coming from YOLOv5 architecture, associated with the object detection theme, were also tested, while for the face points detection task, algorithms were implemented, associated with the keypoint detection theme, which differ from each other in the present Backbone and whose constitutions correspond to variations of CNNs Resnet and HRNetv2. Respectively, the YOLOv5 Small algorithm was chosen (with a precision of $81.86 \%$ ) as well as the algorithm whose Backbone is formed by the Resnet-50 architecture (with a precision of $78.68 \%$ ). These choices, like the mask detection component, were based on the commitment between the obtained metrics and the real-time performance.

Author Contributions: Conceptualization, A.H.J.M. and J.B.; methodology, J.B.; software, C.M. and S.D.; validation, J.B., C.M. and S.D.; formal analysis, J.B., C.M. and S.D.; investigation, C.M. and S.D.; writing - original draft preparation, C.M. and S.D.; writing-review and editing, S.D., C.M. and J.B.; supervision, J.B.; project administration, A.H.J.M. and J.B.; funding acquisition, J.C.F., J.B. and A.H.J.M. All authors have read and agreed to the published version of the manuscript.

Funding: R\&D Project funded by P2020_COVID19, with number 70289.

Institutional Review Board Statement: The study was conducted according to the guidelines of the the Declaration of Helsinki, and approved by the Ethics Committee of Universidade do Minho.

Informed Consent Statement: Written informed consent has been obtained from the patients to publish this paper.

Data Availability Statement: Two datasets [2,3] were published and are available.

Conflicts of Interest: The funders had no role in the design of the study; in the collection, analyses, or interpretation of data; in the writing of the manuscript, or in the decision to publish the results.

\section{References}

1. Cheng, K.K.; Lam, T.H.; Leung, C.C. Wearing face masks in the community during the COVID-19 pandemic: Altruism and solidarity. Lancet 2020. [CrossRef]

2. Melo, C.; Dixe, S.; Fonseca, J.C.; Moreira, A.; Borges, J. MoLa RGB CovSurv. Mendeley Data. V1. Available online: https: / / data.mendeley.com/datasets/vzf939jbxy/1 (accessed on 1 November 2021). [CrossRef]

3. Melo, C.; Dixe, S.; Fonseca, J.C.; Moreira, A.; Borges, J. MoLa IR CovSurv. Mendeley Data. V1. 2021. Available online: https:/ / data.mendeley.com/datasets/rgg6b7tx4s/1 (accessed on 1 November 2021). [CrossRef]

4. Girshick, R.; Donahue, J.; Darrell, T.; Malik, J. Rich feature hierarchies for accurate object detection and semantic segmentation. In Proceedings of the IEEE Conference on Computer Vision and Pattern Recognition (CVPR), Columbus, OH, USA, 23-28 June 2014.

5. Girshick, R. Fast r-CNN. In Proceedings of the IEEE International Conference on Computer Vision (ICCV), Santiago, Chile, 7-13 December 2015.

6. Ren, S.; He, K.; Girshick, R.; Sun, J. Faster r-cnn: Towards real-time object detection with region proposal networks. In Advances in Neural Information Processing Systems 28; Cortes, C., Lawrence, N.D., Lee, D.D., Sugiyama, M., Garnett, R., Eds.; Curran Associates, Inc.: New York, NY, USA, 2016; pp. 91-99.

7. Redmon, J.; Divvala, S.; Girshick, R.; Farhadi, A. You only look once: Unified, real-time object detection. In Proceedings of the IEEE Computer Society Conference on Computer Vision and Pattern Recognition, Las Vegas, NV, USA, 27-30 June 2016; pp. 779-788.

8. Redmon, J.; Farhadi, A. Yolo9000: Better, faster, stronger. In Proceedings of the IEEE Conference on Computer Vision and Pattern Recognition (CVPR), Honolulu, HI, USA, 21-26 July 2017.

9. Redmon, J.; Farhadi, A. YOLOv3: An Incremental Improvement. arXiv 2018, arXiv:1804.02767. 
10. Bochkovskiy, A.; Wang, C.-Y.; Liao, H.-Y.M. YOLOv4: Optimal Speed and Accuracy of object detection. arXiv 2020, arXiv:2004.10934.

11. Ultralytics. Github. 2020. Available online: https:/ / github.com/ultralytics/yolov5 (accessed on 20 November 2021).

12. Jiang, M.; Fan, X.; Yan, H. Retinafacemask: A face mask detector. arXiv 2020, arXiv:2005.03950.

13. Fighting against COVID-19: A Novel Deep Learning Model Based on YOLO-v2 with ResNet-50 for Medical Face Mask Detection I Elsevier Enhanced Reader. Available online: https://reader.elsevier.com/reader/sd/pii/S2210670720308179? token=8284B3488945F354C37E05EC1ADCE9089934FFC31EB0CC7F23948563EEABE22945396983446BBF80DD07EE1AD21127 E9\&originRegion=eu-west-1\&originCreation=20210923103724 (accessed on 3 September 2021).

14. Nagrath, P.; Jain, R.; Madan, A.; Arora, R.; Kataria, P.; Hemanth, J. SSDMNV2: A real time DNN-based face mask detection system using single shot multibox detector and MobileNetV2. Sustain. Cities Soc. 2021, 66, 102692. [CrossRef] [PubMed]

15. Viola, P.; Jones, M. Rapid object detection using a boosted cascade of simple features. In Proceedings of the IEEE Computer Society Conference on Computer Vision and Pattern Recognition, Kauai, HI, USA, 8-14 December 2001. [CrossRef]

16. Li, H.; Lin, Z.; Shen, X.; Brandt, J.; Hua, G. A convolutional neural network cascade for face detection. In Proceedings of the IEEE Computer Society Conference on Computer Vision and Pattern Recognition, Boston, MA, USA, 7-12 June 2015; pp. 5325-5334. [CrossRef]

17. Sun, Y.; Wang, X.; Tang, X. Deep convolutional network cascade for facial point detection. In Proceedings of the 2013 IEEE Conference on Computer Vision and Pattern Recognition, Portland, OR, USA, 23-28 June 2013. [CrossRef]

18. Haavisto, M.; Kaarna, A.; Lensu, L. Deep learning for facial keypoints detection. In Proceedings of the 10th International Conference on Computer Vision Theory and Applications, Berlin, Germany, 11-14 March 2015. [CrossRef]

19. Longpre, S.; Sohmshetty, A. Facial Keypoint Detection. 2016. Available online: https://www.semanticscholar.org/paper/FacialKeypoint-Detection-Longpre-Sohmshetty/8a1ed5e23231e86216c9bdd62419c3b05f1e0b4d (accessed on 3 September 2021).

20. Agarwal, N.; Krohn-Grimberghe, A.; Vyas, R. Facial key points detection using deep convolutional neural network-Naimishnet. arXiv 2017, arXiv:1710.00977.

21. Yang, D.; Yurtsever, E.; Renganathan, V.; Redmill, K.A.; Özgüner, Ü. A Vision-Based Social Distancing and Critical Density Detection System for COVID-19. Sensors 2021, 21, 4608. [CrossRef] [PubMed]

22. Mohammed, M.N.; Hazairin, N.A.; Syamsudin, H.; Al-Zubaidi, S.; Mustapha, S.A.K.S.; Yusuf, E. 2019 Novel Coronavirus Disease (COVID-19): Detection and Diagnosis System Using IoT Based Smart Glasses. Int. J. Adv. Sci. Technol. 2020, 29, 954-960.

23. Hassan, A.; Shahin, I.; Alsabek, M.B. COVID-19 Detection System using Recurrent Neural Networks. In Proceedings of the 2020 International Conference on Communications, Computing, Cybersecurity, and Informatics (CCCI), Sharjah, United Arab Emirates, 3-5 November 2020; pp. 1-5. [CrossRef]

24. Belkacem, A.N.; Ouhbi, S.; Lakas, A.; Benkhelifa, E.; Chen, C. End-to-End AI-Based Point-of-Care Diagnosis System for Classifying Respiratory Illnesses and Early Detection of COVID-19: A Theoretical Framework. Front. Med. 2021, 8, 585578. [CrossRef] [PubMed]

25. Rahman, M.M.; Manik, M.M.H.; Islam, M.M.; Mahmud, S.; Kim, J.-H. An Automated System to Limit COVID-19 Using Facial Mask Detection in Smart City Network. In Proceedings of the 2020 IEEE International IOT, Electronics and Mechatronics Conference (IEMTRONICS), Vancouver, BC, Canada, 9-12 September 2020; pp. 1-5. [CrossRef]

26. Teboulbi, S.; Messaoud, S.; Hajjaji, M.A.; Mtibaa, A. Real-Time Implementation ofAI-Based Face Mask Detection and Social Distancing Measuring System for COVID-19 Prevention. Sci. Program. 2021, 2021, 10589244. [CrossRef]

27. dlib - PyPI. Available online: https:/ / pypi.org/project/dlib / (accessed on 1 June 2021).

28. Liu, Z.; Luo, P.; Wang, X.; Tang, X. Deep learning face attributes in the wild. In Proceedings of the International Conference on Computer Vision (ICCV), Santiago, Chile, 7-13 December 2015.

29. Lin, T.-Y.; Maire, M.; Belongie, S.; Bourdev, L.; Girshick, R.; Hays, J.; Perona, P.; Ramanan, D.; Zitnick, C.L.; Dollár, P. Microsoft coco: Common objects in context. In Proceedings of the 13th European Conference, Zurich, Switzerland, 6-12 September 2014.

30. Le, V.; Brandt, J.; Lin, Z.; Bourdev, L.; Huang, T.S. Interactive facial feature localization. In Proceedings of the 12th European Conference on Computer Vision, Florence, Italy, 7-13 October 2012.

31. Nordstrøm, M.M.; Larsen, M.; Sierakowski, J.; Stegmann, M.B. The Imm Face Database an Annotated Dataset of 240 Face Images. 2004. Available online: http:/ / www.imm.dtu.dk/ aam/aamexplorer/ (accessed on 15 June 2021).

32. Yang, S.; Luo, P.; Loy, C.C.; Tang, X. Wider face: A face detection benchmark. In Proceedings of the IEEE Conference on Computer Vision and Pattern Recognition (CVPR), Las Vegas, NV, USA, 27-30 June 2016.

33. Gallagher, A.; Chen, T. Understanding images of groups of people. In Proceedings of the 2009 IEEE Conference on Computer Vision and Pattern Recognition, Miami, FL, USA, 20-25 June 2009.

34. Chen, S.; Liu, Y.; Gao, X.; Han, Z. Mobilefacenets: Efficient cnns for accurate real-time faceverification on mobile devices. In Proceedings of the 3th Chinese Conference, CCBR 2018, Urumqi, China, 11-12 August 2018.

35. Rizescu, D. Temperature gradient analysis by thermography used in optometry. Int. J. Mechatronics Appl. Mech. 2019, 6, 7-14.

36. FLIR ADK Thermal Vision Automotive Development Kit I Teledyne FLIR. Available online: https://www.flir.eu/products/adk/ (accessed on 13 May 2021).

37. Dhamecha, T.I.; Nigam, A.; Singh, R.; Vatsa, M. Disguise detection and face recognition in visible and thermal spectrums. In Proceedings of the 2013 International Conference on Biometrics (ICB), Madrid, Spain, 4-7 June 2013. 
38. Ghiass, R.S.; Bendada, H.; Maldague, X. Université laval face motion and time-lapse video database (ul-fmtv). In Proceedings of the 14th International Conference on Quantitative Infrared Thermography 2018, Berlin, Germany, 25-29 June 2018.

39. Ibrahim, A.; Gaber, T.; Horiuchi, T.; Snasel, V.; Hassanien, A.E. Human thermal face extraction based on superpixel technique. In Proceedings of the The 1st International Conference on Advanced Intelligent System and Informatics (AISI2015), Beni Suef, Egypt, 28-30 November 2015; Springer: Cham, Switzerland, 2015; Volume 407. [CrossRef]

40. Kong, S.; Heo, J.; Boughorbel, F.; Zheng, Y.; Abidi, B.; Koschan, A.; Yi, M.; Abidi, M. Multiscale fusion of visible and thermal ir images for illumination-invariant face recognition. Int. J. Comput. Vis. 2007, 71, 215-233. [CrossRef]

41. V7Labs. V7-Ai Data Platform for Ml Teams. Available online: https://www.v7labs.com/ (accessed on 25 June 2021).

42. MMPose Contributors. Openmmlab Pose Estimation Toolbox and Benchmark. 2020. Available online: https://github.com/openmmlab/mmpose (accessed on 20 September 2021).

43. Face Mask Detection I Kaggle. Available online: https://www.kaggle.com/andrewmvd/face-mask-detection (accessed on 13 September 2021).

44. Face Mask Detection Dataset I Kaggle. Available online: https://www.kaggle.com/wobotintelligence/face-mask-detectiondataset (accessed on 1 September 2021).

45. Model Face Mask Detection. Available online: https://github.com/AIZOOTech/FaceMaskDetection (accessed on 20 November 2021). 